\title{
Retrofitting infrastructure for skip-stop operation on urban railways
}

\author{
S. M. Oh, K. M. Kim, H. L. Rho \& K. Y. Yang \\ Green Transport and Logistic Institute (Division), \\ Korea Railroad Research Institute, Korea
}

\begin{abstract}
Many urban railways operate a single type normal train without combination with an express train on a line, particularly in Korea. As a result, the average speed of Korean urban trains becomes much slower than those of advanced railways. This paper is one result of Korean research into improving the low speed on urban railways.

This paper presents two case studies of retrofitting urban railway infrastructure for a skip-stop operation; SMRT (Seoul Metropolitan Rapid Transit Co.) and KORAIL (Korea Railroad) lines. The first case takes an approach of minimum investment, and the second case tolerates the required investment for retrofitting infrastructure.

Moreover, it presents several issues for retrofitting the existing infrastructure to make the line enable skip-stop operation. The first is to utilize existing staying sidings for overnight trains in the skip-stop operation. Second is skip-stop scheduling which consists of designing the stop-pattern of express trains and a trade-off between headway versus travel time. Last is a safety issue in the engineering work of an in-operating line and signalling system.
\end{abstract}

Keywords: retrofitting, skip-stop, express train, scheduling.

\section{Introduction}

42 years have passed since the first Korean urban railway service (1974) in the capital area. Recently, more Korean passengers have experienced the advantages of the faster services from KTX (first Korean high-speed railway, 2004) as well as Seoul Metro-9 (first fully-dressed express/normal service urban railway in Korea, 2009). 
Unfortunately, seven urban railways companies (except SeoulMetro-9), who operate 18 lines in the area, operate single all-stop pattern trains without any express trains. Resultantly, an average speed of Korean urban railway becomes much slower than those of advanced railways. This is because most of the lines had been constructed without facilities for skip-stop operation such as passing sidings and high-performance signalling systems to control overtaking time. Now, many passengers, who use the urban railways in the area, want a faster train service from the experience of KTX and SeoulMetro-9.

This paper addresses retrofitting infrastructure to operate express trains together with existing normal trains on the urban railway. Moreover, this paper presents methods and issues of retrofitting the existing infrastructure to make the lines possible to enable skip-stop operation.

However, not a few difficulties exist in retrofitting the infrastructure. Many stations in the lines were constructed underground, which are surrounded by building nearby. Therefore, constructing additional passing sidings to the existing line is fairly difficult. The difficulties can be understood in two major issues amongst many others.

First, daily train operation is possibly affected by the construction work with high possibility. This may cause inconvenience to the citizen, and some of them, particularly who may not benefit from the retrofitting, may blame the retrofitting project.

Second, the investment cost for retrofitting is calculated to be fairly high. KRRI (Korea Railroad Research Institute) have calculated the cost to 150 million dollars for additional construction of a passing siding to an in-operating underground station. The retrofitting cost is quite larger than a cost to be paid at a plan from beginning of construction.

This paper presents two case studies on retrofitting the existing infrastructure in the Korean urban railways; SMRT (Seoul Metropolitan Rapid Transit Co.) and KORAIL (Korea Railroad) lines. The two case studies present overcoming the two major difficulties.

Later of this paper comprised; section two presents a categorization of skipstop schemes and types of infrastructure, section three presents two case studies of SMRT and KORAIL lines, section four presents safety issue on engineering works and technology issue on signalling systems, section five presents conclusions lastly.

\section{Categorization of skip-stop schemes and infrastructure}

This section presents categories both on operation schemes of skip-stop and types of passing sidings. As shown in Fig. 1, schemes of skip-stop operations are categorized into a separated operation and combined one.

The separated operation uses physically separated two rail lines; the one is exclusively for express trains and the other is exclusively for normal trains also. This operation scheme can provide fairly high performance and efficiency in train operation, but the investment for this option is higher than any other schemes. The combined operation allows both express and normal trains be operated in a single 
line. This operation can save investment at the cost of lowered performance and efficiency compare to the physically separated two infrastructures.

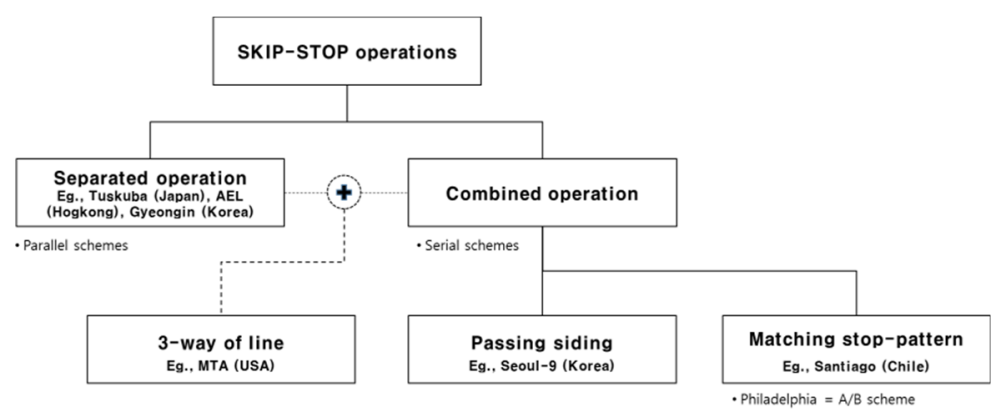

Figure 1: Scheme of the skip-stop operation.

The combined operation can be further categorized into passing sidings and matching stop-pattern schemes more in detail. The scheme of utilizing passing sidings has a normal train refuge in a passing siding for overtaking of an express train. The scheme of matching stop-pattern does not utilize any passing siding contrary to the scheme of passing sidings. In addition, this scheme takes $\mathrm{A} / \mathrm{B}$ pattern of trains instead of express and normal trains. Where $\mathrm{A} / \mathrm{B}$ means two different skip-stop patterns that are designed under consideration of matching between the two patterns mutually complementary.
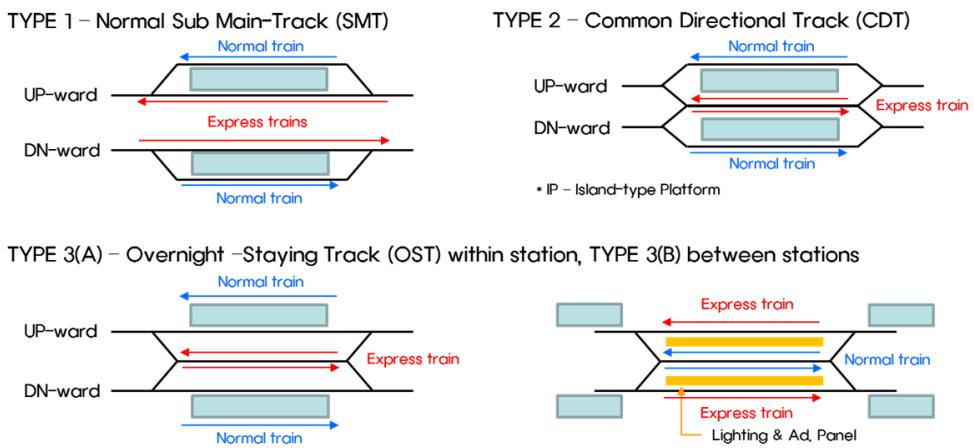

TYPE 4 - Crossover Track
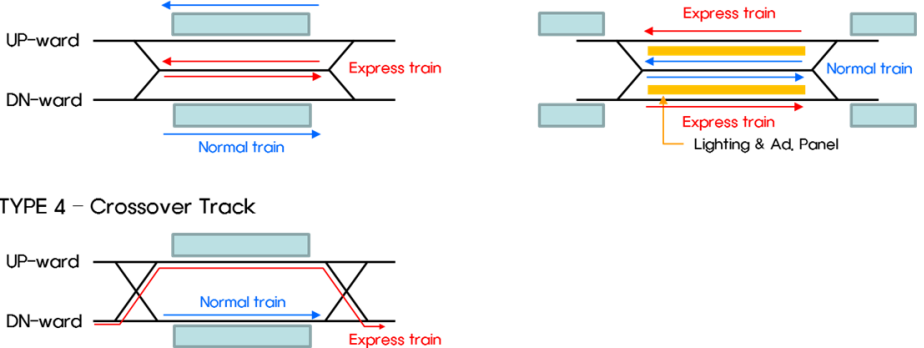

Figure 2: Types of passing sidings. 
On the other hand, the scheme of 3-way of lines takes advantages of both separated and combined operation. This scheme of operation is applied to the New York Metro.

Fig. 2 shows types of passing sidings. Amongst the 5 types, TYPE 4 is not used in practice Korean urban railway. In TYPE 4, a normal train shall wait at the main track for passing an express train through the opposite direction of the track. Authors could not find any case of utilizing TYPE 4 in the world for ordinary skipstop operation. Actually, TYPE 4 was created by author's imagination.

Contrary to TYPE 4, TYPE 1 is one of the most common types of passing sidings. In Korea, a passing siding is also referred to a sub-main tack (SMT). A normal train shall wait at the sub-main track for refuging an express train through the main track.

Korean railway guidelines conceptually differentiate the SMT from the siding. Korean engineers regard the SMT as a type of the main track, not as a siding. Thus, they believe an SMT is used for commercial train operation such as refuging normal trains for passing express trains but siding is used only for accommodating overnight or maintenance trains after an end of a daily commercial operation. Korean guidelines permit lower number of a switch (\#8) for a siding than for an SMT (\#10 or \#12). Practically, the SMT (referred in Korea) corresponds to the passing siding (referred in international), so that this paper will use the terminology of 'passing siding' instead of SMT in order to avoid confusing in understanding.

TYPE 2 has a common directional track (CDT) with which an express shall pass through between two (up/down) directional tracks. The CDT can be utilized for various aims; staying overnight trains and maintenance vehicles besides passing express trains. One demerit of the type is what length of the rail tracks at the front and end points shall be extended due to place a platform between the directional and common directional tracks.

TYPE 3 is further differentiated into two different forms; TYPE 3(A) and TYPE 3(B). A track lies between two main tracks in TYPE 3 has been used only for accommodating overnight or maintenance trains, not used for passing express trains. TYPE 3(A) places an overnight-stay track (OST) inside of a station, but TYPE 3(B) does it outside of a station. Both of the two types of TYPE 3 have been applied in designing Korean urban railways not seldom.

\section{Case studies}

\subsection{An SMRT line}

The SMRT line has 33 stations in $35 \mathrm{~km}$ approximately. Each train stops at every station in the line without any skip. The travel time of the line is amount to 57 minutes. As Fig. 3 shows, one end of the line forms a loop and the other end facilitates single depot for rail vehicles. The single depot has insufficient space to accommodate whole rail vehicles of the line. Instead, 5 OSTs are facilitated to accommodate overnight and maintenance trains. 


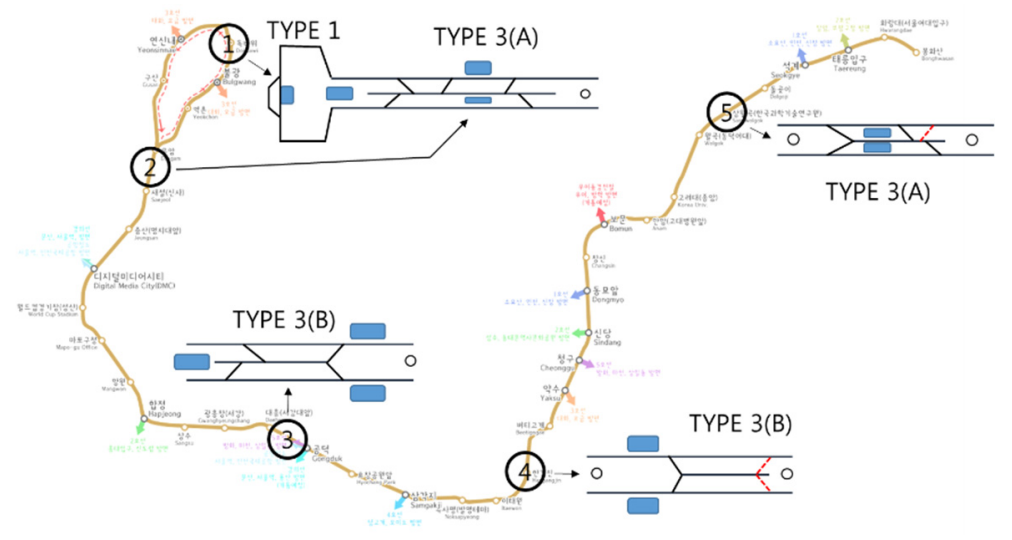

Figure 3: A case of SMRT line.

A key concept, in this case, is to provide express service together with existing normal service with minimum investment to retrofit infrastructure. We carried out engineering study to improve the five OST to passing siding (SMT) carefully.

OST (1) lies in a single line loop section is slightly different from TYPE 1 in a position of a platform. As the platform positioned on the main track side, a normal train should stop on the main track and an express train should overtake the normal train through the side track. To prevent from reducing through-speed of the express train, we checked changing switch from \#8 to \#10 and \#12 facilitated in the side track. In addition, a rail cant and a rail width were carefully checked to run the curved side track at a higher speed than before.

OST (2) is classified to TYPE 3(A) besides only a platform positioned outside of the main track. Due to the position of the platform, a normal train stop on the main track and an express train overtakes the normal train through side track similarly to OST (1). Every switch was checked to improve from \#8 to \#10/\#12.

OST (3) is classified to TYPE 3(B), so that an express and normal trains meet at a middle of consecutive two stations. When a normal train waits in a middle of the two stations, passengers' discomfort should be alleviated by lighting in the OST.

OST (4) can be classified to TYPE 3(B), but the OST is not connected to the main track at the right side as shown in the dashed lines. To have a normal train refuge in the OST, and then turn back to the main track, switches should be added to the point of the dashed line. Unfortunately, the main track sharply curved near the dashed position, so that facilitating additional switch was not easy. Inevitably, the switches were designed to place more left side from the dashed point, then the side track was designed to much shorter than required length for train length and over-run margin. To overcome the shortage of over-run margin, we planned a normal train to stop on the main track and an express train overtakes the normal train through the side track. When a normal train stops at the main track, two 
switches in both sides were locked in the open mode to achieve required over-run margin.

OST (5) is classified to TYPE 3(A). A switch needs to add at the dashed line. However, one difficulty to add a switch is existing of pillars enduring weight over a tunnel at the position. In addition, the interval between OST (4) and OST (5) is much larger than the others, which enlarges average headway. Thus, we restricted the spatial scope of skip-stop operation to OST (1)-OST (4), and gave up OST (5).
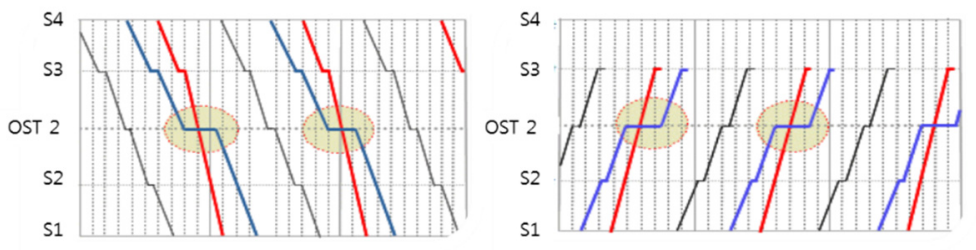

Figure 4: A skip-stop schedule of SMRT line

Fig. 4 shows a skip-stop schedule around OST (2) of the case line. A frequency ratio of express and normal trains is $\mathrm{E}: \mathrm{N}=1: 2$. By using this skip-stop operation scheme, travel time between OST (1)-OST (4) (a half of total kilometres) is cut down by 10 minutes. We estimated a benefit - cost analysis yielded $\mathrm{B} / \mathrm{C}=11.0$ approximately.

\subsection{A KORAIL line}

The KORAIL line has 36 stations in $53 \mathrm{~km}$ approximately. This line has partially operated express trains together with normal trains. As shown in Fig. 5, 6 express trains operate in skip-stop mode in S1-S13 currently and operate in an all-stop mode in the other section. An average speed of the express train gets to $53.3 \mathrm{~km} / \mathrm{h}$ in the skip-stop section, but fall down to $37.1 \mathrm{~km} / \mathrm{h}$ in the other section. The six express trains run in $76 \mathrm{~min}$., but all the other normal trains run in $85.5 \mathrm{~min}$. over the whole line.

This case aims at extending skip-stop section to S14-S36 too. This case has no OST in the line so that required passing siding should be newly constructed. We decided a type of the new passing siding to TYPE 1, same to the type of the existing passing siding.

Deciding the positions of passing sidings, their consecutive distance should be equivalent as far as possible. However, the equivalent positions should meet engineering conditions. This paper presents five engineering conditions to be met by a new passing siding. Particularly, the conditions were set up under consideration of retrofitting existing infrastructure, not of constructing absolutely new. 


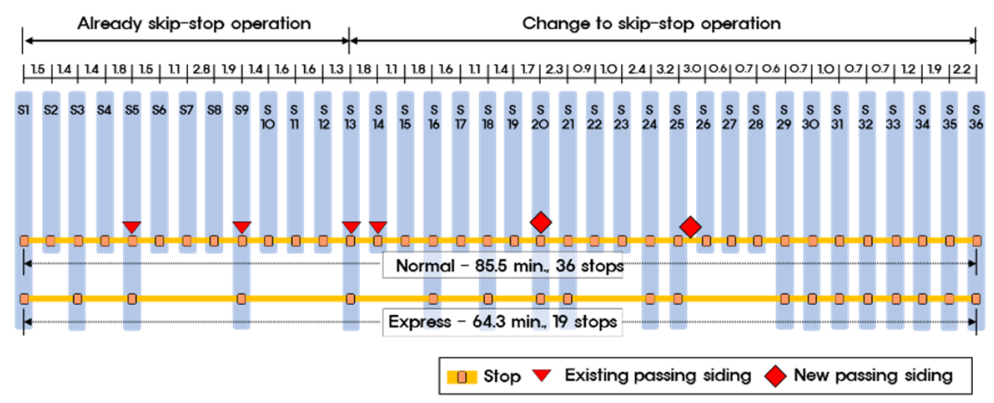

Figure 5: A case of the KORAIL line.

- Minimum width possible to extend for building new passing sidings (11.0m);

- Maximum gradient for the passing siding (15/1000);

- Minimum length of non-curved main track to facilitate switch (\#10\#12, 47.9m-77.9m);

- Minimum length for accommodating refuge train and over-run;

- Existence of building on the ground.

We scrutinized the five conditions over all stations in S14-S36 and found 2 positions not only meeting the conditions but spacing equivalent between them. The first one is in S20 and the other one between S25-S26. At first, we found the second position in $\mathrm{S} 25$, but retrofitting infrastructure in $\mathrm{S} 25$ requires much higher cost than the first one. Thus, we moved the second position to the middle of S25 and S26. The second passing siding should facilitate a lighting equipment as OST (3) in the case of SMRT line.

In this case study, the new express train can cut down total travel time by 12 min. comparing to the 6 existing express trains, and by $21 \mathrm{~min}$. comparing to the normal trains. We estimated an average reduction of travel time get to $2.5 \mathrm{~min}$ for individual passenger and benefit-cost analysis yielded $\mathrm{B} / \mathrm{C}=2.7$ approximately.

\section{Skip-stop scheduling}

\subsection{Stop pattern of an express train}

One of the simplest principles to select stops of an express train is traffic volume since passengers hope express trains stop at stations near their home and office. Fig. 6 shows traffic volume of the SMRT line. We can put on a reference level to the Fig. 6. If we want, we can adjust the level, then select stations whose traffic volume exceed the level. Another point, to select express stops, is to consider whether a station is transfer one or not.

However, traffic volume is not everything to be considered in designing a stoppattern of an express train. The main purpose of retrofitting is to provide faster train service to reduce travel time. Actually, a speed of normal train does not change between before and after retrofitting, but the reduction of travel time is 
achieved by introducing express trains. Thus, a design of express stop pattern makes a direct effect on a reduction of travel time, since the more the train stops at stations, the slower the train speed.

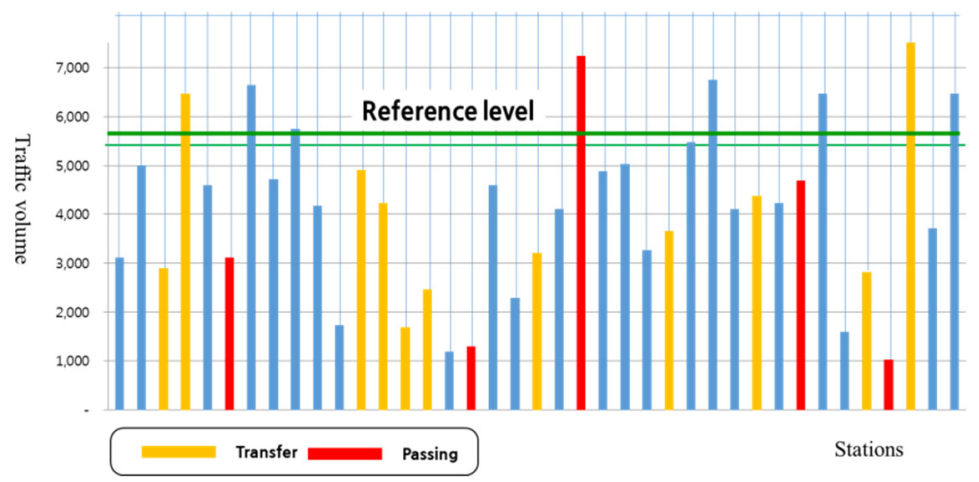

Figure 6: Traffic volume of SMRT line.

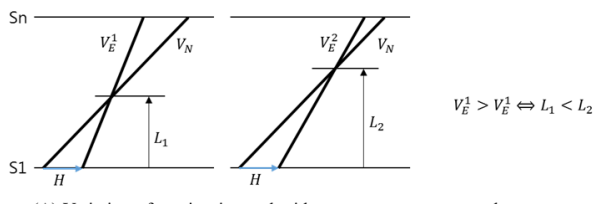

(A) Variation of passing interval with respect to express speed
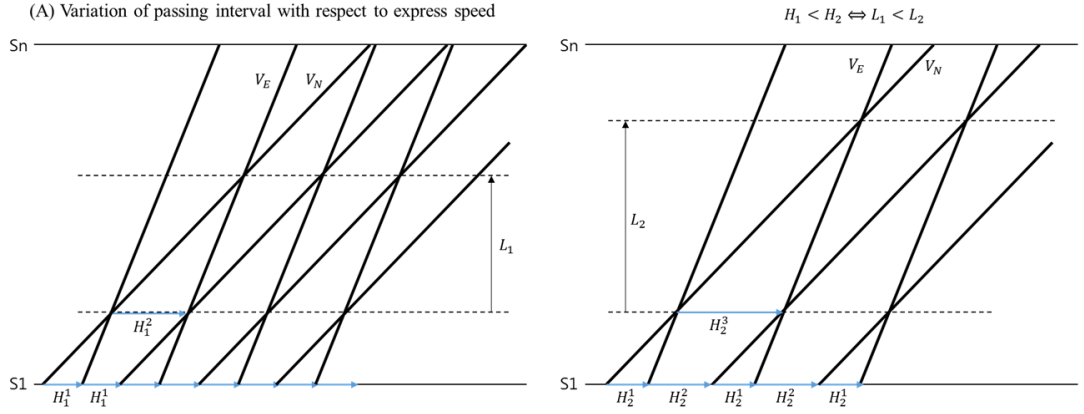

(B) Variation of headway with respect to passing interval

Figure 7: Relationship between passing interval, express speed and headway.

Fig. 7 shows a relationship between the 'interval of passing siding', 'speed of express train' and average headway. Fig. 7(A) shows that an express train (E1) runs faster than the other express train (E2), this means the number of stops of E2 is larger than E1, then an interval between passing sidings for E1 is smaller than E2 under conditions of same headway $(\mathrm{H})$ and normal speed $\left(V_{N}\right)$. Fig. 7(B) shows that an interval between passing sidings $\left(L_{1}\right)$ is shorter than the other $\left(L_{2}\right)$, then average headway of the former case $\left(H_{1}\right)$ is shorter than the later $\left(H_{2}\right)$ under conditions of same normal $\left(V_{N}\right)$ and express $\left(V_{E}\right)$ speeds. 
We can inference that the number of express stops should be decided under consideration of passing interval and service headway.

\subsection{Skip-stop scheduling by Dr.METRO}

Even though, this paper addresses the basic principle to decide a stop-pattern of an express train. It is still quite difficult to find an optimal stop-pattern and skipstop scheduling since the intervals of passing sidings are not identical in practice. The problem is solved by Dr.METRO scheduling software $[1,2]$.

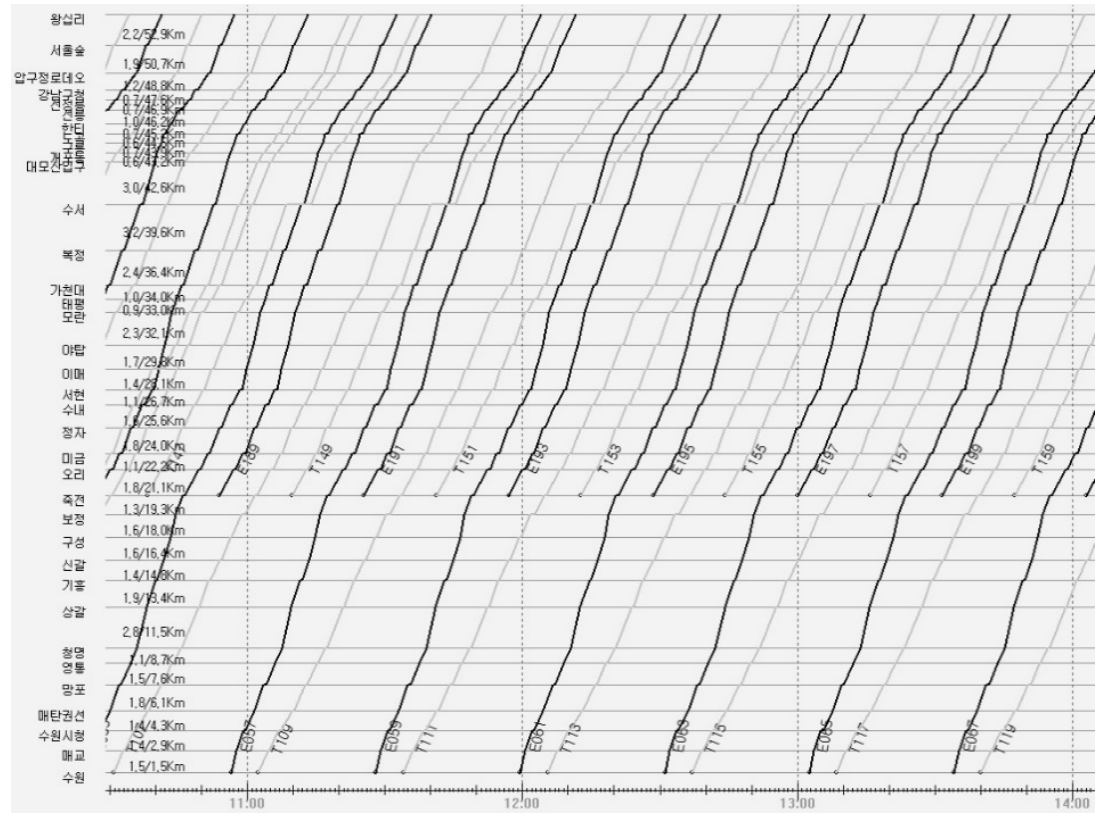

Figure 8: A skip-stop schedule by Dr.METRO.

Fig. 8 shows a skip-stop schedule planned by Dr.METRO for a case of KORAIL line. Dr.METRO can adjust headway and speeds of express and normal trains to harmonize their meet-pass operations at decided positions of passing sidings.

\section{Engineering works and signalling systems}

\subsection{Engineering works with uninterrupted operation}

Major engineering work is to add a new passing siding in an underground tunnel in retrofitting infrastructure of an urban railway for skip-stop operation. This a new passing siding is constructed without interruption of commercial operation as far as possible. This requires a safe-granted method of engineering work. 
Most underground tunnels in an urban railway are classified into two types; the one is an open-cut box and the other is NATM tunnels, at least in Korea. The methods of engineering work should be selected properly for the two classes.

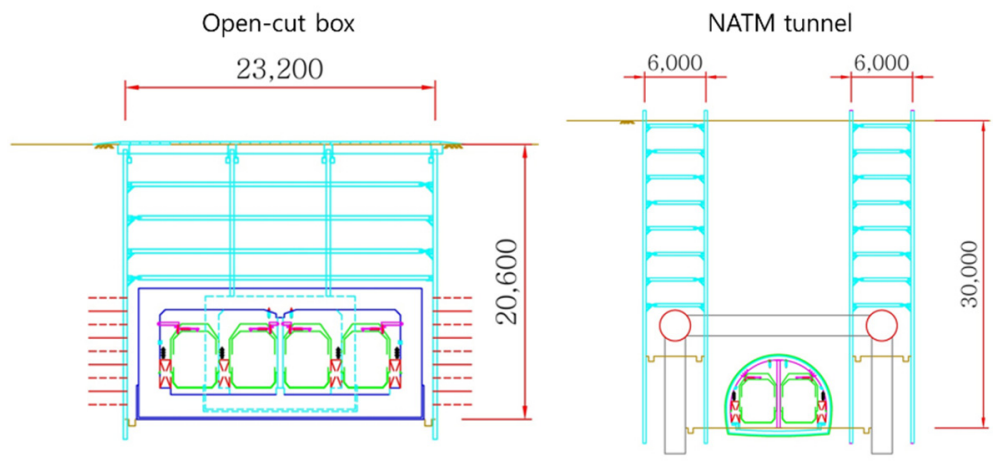

Figure 9: Engineering works for open-cut box and NATM tunnel.

Fig. 9 shows methods of engineering works for the two types of a tunnel [3]. As shown in the figure, the open-cut box cannot be used where a depth is high, only NATM tunnel is possible.

The engineering work for the NATM tunnel is more difficult than the open-cut box. First, excavate in both sides of the tunnel, then facilitate a steel structure to bear earth pressure from up and sides. Second, remove soil, add new passing siding inside of the structure. Last, remove a wall of the tunnel and move a catenary system to the structure.

\subsection{Modification of signalling systems}

One of most difficult step in the case of the KORAIL line is the engineering work in the NATM tunnel, but the one in the case of SMRT line is a modification of signalling systems.

To newly launch a skip-stop operation scheme, modification of signalling systems (e.g. ATO and ATC) is indispensable. A large portion of an industry of railway signalling system is controlled by a small number of international companies. Most Korean urban railways selected their signalling systems from the international companies too.

Thus, the urban railways may request for cooperation to the companies for launching the new skip-stop operation. Otherwise, they cannot help but substitute the existing systems by using their own systems completely. However, this case results in cost increased inevitably.

\section{Conclusions}

This paper addresses two case studies of retrofitting infrastructure for skip-stop operation. Several issues are presented including skip-stop scheduling and engineering works to be treated carefully. 
We guess many international urban railways might have selected an operation scheme like previous Korean urban railways. However, we started research to change the operation scheme by retrofitting infrastructure. The two case studies showed prospective estimations in economic benefits as well as operational performance.

Another thing, we learned from the two case studies, is a skip-stop operation scheme should be considered at the beginning of construction plan of a new line. This might save investment cost than retrofitting later than opening a single type operation.

\section{References}

[1] Oh, S. M. \& Min, J. H., Dr.METRO: a demand-responsive metro-train operation planning program, Computers in Railways XIV (2014), pp. 595601.

[2] Oh, S. M., Improvements of Dr.METRO and its application to urban railway scheduling, RAILTOKYO 2015.

[3] Korea Railroad Research Institute internal report, 2013. 